\title{
If cM1, specify site(s)
}

National Cancer Institute

\section{Source}

National Cancer Institute. If CM1, specify site(s). NCI Thesaurus. Code C157098.

A directive to indicate the anatomic sites if metastasis is categorized as CM1. 